\title{
On non-Newtonian measure for $\alpha$-closed sets
}

\author{
Ŏguz Ŏgur ${ }^{1}$ and Sezgin Demir ${ }^{2}$ \\ ${ }^{1}$ Giresun University, Faculty of Education, Department of Elementary Mathematics, Giresun, Turkey \\ ${ }^{2}$ Giresun University, Art and Science Faculty, Department of Mathematics, Giresun, Turkey
}

Received: 19 February 2019, Accepted: 21 May 2019

Published online: 27 May 2019.

Abstract: In this paper, we define the non-Newtonian measure for $\alpha$-closed sets and study on its some basic properties.

Keywords: Non-Newtonian measure, non-Newtonian series, geometric calculus.

\section{Introduction}

Non-Newtonian calculus was created by Katz and Grossman as an alternative to classic calculus between 1967-1970 [1]. The first arithmetic calculus is defined as geometric, harmonic and quadratic calculus. Grossman also studied some properties of derivatives and integrals in non-Newtonian calculus [2]. Bashirov et. al. have recently studied some basic properties of derivatives and integrals in multiplicative calculus and gave the results with applications [3]. Later, Duyar, Sağır and Oğur gave some basic topological properties of non- Newtonian calculus [4]. Recently, Duyar and Sağır [5] introduced the concepts of the non-Newtonian measure for open sets. For more details see [7], [8], [9], [10].

Let $\alpha$ be a generator, $\alpha$ is a one-to-one function whose domain is real numbers and whose range is a subset $A$ of $\mathbb{R}$. We know that each generator produces exatly one arithmetic and conversely, each arithmetic is produced by one generator. For instance, the identity function $I$ generates the classic arithmetic and the exponential function exp generates geometric arithmetic. Let take a generator $\alpha$ such that have the following basic algebraic operations $[1-5]$ :

$$
\begin{array}{lc}
\alpha \text {-addition } & x \dot{+} y=\alpha\left\{\alpha^{-1}(x)+\alpha^{-1}(y)\right\} \\
\alpha \text {-subtraction } & x \dot{-} y=\alpha\left\{\alpha^{-1}(x)-\alpha^{-1}(y)\right\} \\
\alpha \text {-multiplicative } & x \dot{\times} y=\alpha\left\{\alpha^{-1}(x) \times \alpha^{-1}(y)\right\} \\
\alpha \text {-division } & x / y=\alpha\left\{\alpha^{-1}(x) / \alpha^{-1}(y)\right\} \\
\alpha \text {-order } & x \leq y \Leftrightarrow \alpha^{-1}(x) \leq \alpha^{-1}(y)
\end{array}
$$

for every $x, y \in A$.

The set of non-Newtonian numbers is defined as $\mathbb{R}(N)=\{\alpha(x): x \in \mathbb{R}\}$. A $\alpha$ - closed interval on $\mathbb{R}(N)$ can be represented by

$$
[a, b]_{N}=\{x \in \mathbb{R}(N): a \dot{\leq} x \leq b\}=\left\{x \in \mathbb{R}(N): \alpha^{-1}(a) \leq \alpha^{-1}(x) \leq \alpha^{-1}(b)\right\}=\alpha\left\{\left[\alpha^{-1}(a), \alpha^{-1}(b)\right]\right\}
$$

Definition 1. Let $F$ and $S$ be two point sets. If $F \subset S$, then the set $S-F$ is called to complement of the set $F$ with respect to the set $S$ and denoted by the symbol $C_{S}^{F}$. 
Theorem 1. Let $F$ be a non-void bounded $\alpha$-closed set and let $S$ be the smallest $\alpha$-closed interval containing the set $F$. Then the set $C_{S}^{F} \alpha$-open [5].

Definition 2. The measure $m_{N}(a, b)_{N}$ in $\mathbb{R}(N)$ is defined by

$$
m_{N}(a, b)_{N}=\alpha\left\{m\left(\alpha^{-1}(a), \alpha^{-1}(b)\right)\right\}
$$

[5].

Definition 3. The measure $m_{N} G$ of a non-void bounded open set $G$ in $\mathbb{R}(N)$ is the sum of the measures of all its component intervals $\delta_{k}$ :

$$
m_{N} G={ }_{N} \sum_{k} m_{N} \delta_{k}
$$

Here it should be noted that

$$
m_{N} G={ }_{N} \sum_{k} m_{N}\left(a_{k}, b_{k}\right)_{N}={ }_{N} \sum_{k} b_{k} \dot{-} a_{k}
$$

where $\delta_{k}=\left(a_{k}, b_{k}\right)_{N}[5]$.

Theorem 2. Let $G_{1}$ and $G_{2}$ be two bounded open set in $\mathbb{R}(N)$. If $G_{1} \subset G_{2}$, then

$$
m_{N} G_{1} \leq m_{N} G_{2}
$$

[5].

In this paper, we define and study on non-Newtonian measure of bounded closed sets as a generalization of known results in real analysis.

\section{Main results}

Definition 4. In $\mathbb{R}(N)$, the measure of a non-void bounded $\alpha$-closed set $F$ is defined as follows

$$
m_{N} F=\alpha\left\{m\left(\alpha^{-1}(A), \alpha^{-1}(B)\right)-m\left(\alpha^{-1}\left(C_{S}^{F}\right)\right)\right\}
$$

where $S=[A, B]_{N}$ is the smallest $\alpha$-closed interval containing the set $F$.

We can restate the above relation as follows; since $C_{S}^{F}$ is a $\alpha$-open set, it can be written in the form $C_{S}^{F}=\cup_{k}\left(a_{k}, b_{k}\right)_{N}$. Thus, we get

$$
\begin{aligned}
m_{N} F & =\alpha\left\{m\left(\alpha^{-1}(A), \alpha^{-1}(B)\right)-m\left(\alpha^{-1}\left(C_{S}^{F}\right)\right)\right\} \\
& =\alpha\left\{m\left(\alpha^{-1}(A), \alpha^{-1}(B)\right)-m\left(\alpha^{-1}\left(\cup_{k}\left(a_{k}, b_{k}\right)_{N}\right)\right)\right\} \\
& =\alpha\left\{m\left(\alpha^{-1}(A), \alpha^{-1}(B)\right)-m\left(\cup_{k}\left(\alpha^{-1}\left(a_{k}\right), \alpha^{-1}\left(b_{k}\right)\right)\right)\right\} \\
& =\alpha\left\{m\left(\alpha^{-1}(A), \alpha^{-1}(B)\right)-\sum_{k}\left(\alpha^{-1}\left(b_{k}\right)-\alpha^{-1}\left(a_{k}\right)\right)\right\} \\
& =\alpha\left\{\alpha^{-1}(B)-\alpha^{-1}(A)-\sum_{k}\left(\alpha^{-1}\left(b_{k}\right)-\alpha^{-1}\left(a_{k}\right)\right)\right\} \\
& =\alpha \alpha^{-1}\left(\alpha\left(\alpha^{-1}(B)-\alpha^{-1}(A)\right)\right)-\alpha^{-1}\left(\alpha\left(\sum_{k} \alpha^{-1}\left(\alpha\left(\alpha^{-1}\left(b_{k}\right)-\alpha^{-1}\left(a_{k}\right)\right)\right)\right)\right)
\end{aligned}
$$




$$
\begin{aligned}
& =\alpha\left\{\alpha^{-1}(B \dot{-} A)-\alpha^{-1}\left({ }_{N} \sum_{k} \alpha\left(\alpha^{-1}\left(b_{k}\right)-\alpha^{-1}\left(a_{k}\right)\right)\right)\right\} \\
& =\alpha\left\{\alpha^{-1}(B \dot{-} A)-\alpha^{-1}\left({ }_{N} \sum_{k} m_{N}\left(a_{k}, b_{k}\right)_{N}\right)\right\} \\
& =\alpha\left\{\alpha^{-1}(B-A)-\alpha^{-1}\left(m_{N} C_{S}^{F}\right)\right\} \\
& =B-A-m_{N}\left(C_{S}^{F}\right)
\end{aligned}
$$

Remark. If $F=[a, b]_{N}$, then $S=[a, b]_{N}$ and $C_{S}^{F}=\varnothing$, so that $m_{N} F=b-a$. If $F$ is the union of a finite number of pairwise disjoint closed intervals in $\mathbb{R}(N)$, namely $F=\left[a_{1}, b_{1}\right]_{N} \cup\left[a_{2}, b_{2}\right]_{N} \cup \ldots \cup\left[a_{n}, b_{n}\right]_{N}$, then $m_{N} F={ }_{N} \sum_{k=1}^{n} b_{k} \dot{-} a_{k}$.

Example 1. Let take geometric calculus and let $F=\left[a_{1}, b_{1}\right]_{N} \cup\left[a_{2}, b_{2}\right]_{N}$. Then, we have $S=\left[a_{1}, b_{2}\right]_{N}$ and $C_{S}^{F}=\left(b_{1}, a_{2}\right)_{N}$. Thus, the measure of $\alpha$-closed set is

$$
m_{N} F=\exp \left\{\left(\ln b_{2}-\ln a_{1}\right)-\left(\ln a_{2}-\ln b_{1}\right)\right\}=\exp \left\{\ln \frac{b_{2} b_{1}}{a_{1} a_{2}}\right\}=\frac{b_{1} b_{2}}{a_{1} a_{2}} .
$$

Theorem 3. The non-Newtonian measure of a bounded $\alpha$-closed set $F$ is non-negative.

Proof. Let $F$ be a bounded $\alpha$-closed set and let $S=[a, b]_{N}$ be the smallest $\alpha$-closed interval containing the set $F$.Then

$$
m_{N} F=\alpha\left\{m\left(\alpha^{-1}(a), \alpha^{-1}(b)\right)-m\left(\alpha^{-1}\left(C_{S}^{F}\right)\right)\right\}=\alpha\left\{\alpha^{-1}(b)-\alpha^{-1}(a)-m\left(\alpha^{-1}\left(C_{S}^{F}\right)\right)\right\}>\alpha(\dot{0}) .
$$

Lemma 1. Let $F$ be a bounded $\alpha$-closed set and let $\Delta$ be an $\alpha$-open interval containing $F$. Then $m_{N} F=m_{N} \Delta-m_{N}$ $\left(C_{\Delta}^{F}\right)$.

Proof. Let $\Delta=(A, B)_{N}$ and let $S=[a, b]_{N}$ be the smallest $\alpha$-closed interval containing the set $F$. Then, we have

$$
\begin{aligned}
m_{N} F & =\alpha\left\{m\left(\alpha^{-1}(a), \alpha^{-1}(b)\right)-m\left(\alpha^{-1}\left(C_{S}^{F}\right)\right)\right\} \\
& =\alpha\left\{\alpha^{-1}(b)-\alpha^{-1}(a)-m\left(\alpha^{-1}\left(C_{S}^{F}\right)\right)\right\} \\
& =\alpha\left\{\alpha^{-1}(B)-\alpha^{-1}(A)-m\left(\alpha^{-1}\left(C_{\Delta}^{F}\right)\right)\right\} \\
& =\alpha\left\{\alpha^{-1}(B)-\alpha^{-1}(A)-\alpha^{-1}\left(\alpha\left(m\left(\alpha^{-1}\left(C_{\Delta}^{F}\right)\right)\right)\right)\right\} \\
& =\alpha\left\{\alpha^{-1}(B)-\alpha^{-1}(A)-\alpha^{-1}\left(m_{N}\left(C_{\Delta}^{F}\right)\right)\right\} \\
& =B \dot{-}-m_{N}\left(C_{\Delta}^{F}\right) \\
& =m_{N} \Delta-m_{N}\left(C_{\Delta}^{F}\right) .
\end{aligned}
$$

Theorem 4. Let $F_{1}$ and $F_{2}$ be two non-void bounded $\alpha$-closed sets in $\mathbb{R}(N)$. If $F_{1} \subset F_{2}$, then $m_{N} F_{1} \leq m_{N} F_{2}$.

Proof. Let $S=(a, b)_{N}$ be an $\alpha$-open interval containing the set $F_{2}$. We can easily see that

$$
\begin{aligned}
m_{N} F_{1} & =\alpha\left\{m\left(\alpha^{-1}(a), \alpha^{-1}(b)\right)-m\left(\alpha^{-1}\left(C_{S}^{F_{1}}\right)\right)\right\} \\
& =\alpha\left\{\alpha^{-1}(b)-\alpha^{-1}(a)-m\left(\alpha^{-1}\left(C_{S}^{F_{1}}\right)\right)\right\} \\
& \leq \alpha\left\{\alpha^{-1}(b)-\alpha^{-1}(a)-m\left(\alpha^{-1}\left(C_{S}^{F_{2}}\right)\right)\right\} \\
& =\alpha\left\{m\left(\alpha^{-1}(a), \alpha^{-1}(b)\right)-m\left(\alpha^{-1}\left(C_{S}^{F_{2}}\right)\right)\right\} \\
& =m_{N} F_{2} .
\end{aligned}
$$


Theorem 5. Let $F$ be an $\alpha$-closed set and let $G$ be a bounded $\alpha$-open set in $\mathbb{R}(N)$. If $F \subset G$, then $m_{N} F \leq m_{N} G$.

Proof. Let $S=(a, b)_{N}$ be an $\alpha$-open interval containing the set $G=\cup_{k}\left(a_{k}, b_{k}\right)_{N}$. We can easily see that

$$
\begin{aligned}
m_{N} F & =\alpha\left\{m\left(\alpha^{-1}(a), \alpha^{-1}(b)\right)-m\left(\alpha^{-1}\left(C_{S}^{F}\right)\right)\right\} \\
& =\alpha\left\{\alpha^{-1}(b)-\alpha^{-1}(a)-m\left(\alpha^{-1}\left(C_{S}^{F}\right)\right)\right\} \\
& \leq \alpha\left\{\sum_{k}\left(\alpha^{-1}\left(b_{k}\right)-\alpha^{-1}\left(a_{k}\right)\right)\right\} \\
& ={ }_{N} \sum_{k} \alpha\left(\alpha^{-1}\left(b_{k}\right)-\alpha^{-1}\left(a_{k}\right)\right) \\
& ={ }_{N} \sum_{k} b_{k}-a_{k} \\
& =m_{N} G .
\end{aligned}
$$

Theorem 6. The non-Newtonian measure of a bounded $\alpha$-open set $G$ is the least upper bound of the mesure of all $\alpha$-closed sets contained in $G$.

Proof. By the preceding theorem, $m_{N} G$ is an upper bound for the measures of $\alpha$-closed sets $F \subset G$. Let $G=\bigcup_{k}\left(\lambda_{k}, \mu_{k}\right)_{N}$. Since $m_{N} G={ }_{N} \sum_{k} \mu_{k} \dot{-} \lambda_{k}$, we have

$$
\begin{aligned}
\alpha^{-1}\left(m_{N} G\right) & =\alpha^{-1}\left(N \sum_{k} \mu_{k}-\lambda_{k}\right) \\
& =\alpha^{-1}\left(\alpha\left(\sum_{k} \alpha^{-1}\left(\mu_{k}-\lambda_{k}\right)\right)\right) \\
& =\sum_{k} \alpha^{-1}\left(\mu_{k} \dot{-} \lambda_{k}\right) \\
& =\sum_{k} \alpha^{-1}\left(\alpha\left(\alpha^{-1}\left(\mu_{k}\right)-\alpha^{-1}\left(\lambda_{k}\right)\right)\right) \\
& =\sum_{k} \alpha^{-1}\left(\mu_{k}\right)-\alpha^{-1}\left(\lambda_{k}\right) .
\end{aligned}
$$

Take an arbitrary $\varepsilon>\dot{0}$ and find a natural number $n$ so large that

$$
\sum_{k=1}^{n} \alpha^{-1}\left(\mu_{k}\right)-\alpha^{-1}\left(\lambda_{k}\right)>\alpha^{-1}\left(m_{N} G\right)-\frac{\alpha^{-1}(\varepsilon)}{2}
$$

Therefore, we have

$$
\alpha^{-1}\left(\alpha\left(\sum_{k=1}^{n} \alpha^{-1}\left(\mu_{k}\right)-\alpha^{-1}\left(\lambda_{k}\right)\right)\right)>\alpha^{-1}\left(\alpha\left(\alpha^{-1}\left(m_{N} G\right)-\frac{\alpha^{-1}(\varepsilon)}{2}\right)\right)
$$

and so

$$
\alpha\left(\sum_{k=1}^{n} \alpha^{-1}\left(\mu_{k}\right)-\alpha^{-1}\left(\lambda_{k}\right)\right)>\alpha\left(\alpha^{-1}\left(m_{N} G\right)-\frac{\alpha^{-1}(\varepsilon)}{2}\right)
$$

which gives

$$
N \sum_{k=1}^{n}=\mu_{k} \dot{-} \lambda_{k}>m_{N} G-\frac{\varepsilon}{\dot{2}}
$$


For every $k(k=1,2, \ldots, n)$, we choose a $\alpha$-closed inerval $\left[a_{k}, b_{k}\right]_{N}$ so that $\left[a_{k}, b_{k}\right]_{N} \subset\left(\lambda_{k}, \mu_{k}\right)_{N}$. Thus, we get

$$
\alpha\left[\alpha^{-1}\left(a_{k}\right), \alpha^{-1}\left(b_{k}\right)\right] \subset \alpha\left(\alpha^{-1}\left(\lambda_{k}\right), \alpha^{-1}\left(\mu_{k}\right)\right)
$$

and so

$$
\left[\alpha^{-1}\left(a_{k}\right), \alpha^{-1}\left(b_{k}\right)\right] \subset\left(\alpha^{-1}\left(\lambda_{k}\right), \alpha^{-1}\left(\mu_{k}\right)\right)
$$

Therefore, we have

$$
\alpha^{-1}\left(b_{k}\right)-\alpha^{-1}\left(a_{k}\right)>\alpha^{-1}\left(\mu_{k}\right)-\alpha^{-1}\left(\lambda_{k}\right)-\frac{\alpha^{-1}(\varepsilon)}{2 n}
$$

and so

$$
\alpha^{-1}\left(\alpha\left(\alpha^{-1}\left(b_{k}\right)-\alpha^{-1}\left(a_{k}\right)\right)\right)>\alpha^{-1}\left(\alpha\left(\alpha^{-1}\left(\mu_{k}\right)-\alpha^{-1}\left(\lambda_{k}\right)-\frac{\alpha^{-1}(\varepsilon)}{2 n}\right)\right) .
$$

Then, we get, by inequlity above

$$
\alpha\left(\alpha^{-1}\left(b_{k}\right)-\alpha^{-1}\left(a_{k}\right)\right)>\alpha\left(\alpha^{-1}\left(\mu_{k}\right)-\alpha^{-1}\left(\lambda_{k}\right)-\frac{\alpha^{-1}(\varepsilon)}{2 n}\right)
$$

which means

$$
m_{N}\left[a_{k}, b_{k}\right]_{N}>m_{N}\left(\lambda_{k}, \mu_{k}\right)_{N} \dot{-} \frac{\varepsilon}{\alpha(2 n)} .
$$

Let define $F_{0}=\bigcup_{k=1}^{n}\left[a_{k}, b_{k}\right]_{N}$. It is clear that $F_{0} \subset G$ and $F_{0}$ is $\alpha$-closed set.

Thus, we have

$$
m_{N} F_{0}={ }_{N} \sum_{k=1}^{n} b_{k} \dot{-} a_{k}=\alpha\left\{\sum_{k=1}^{n} \alpha^{-1}\left(b_{k}-a_{k}\right)\right\}=\alpha\left\{\sum_{k=1}^{n} \alpha^{-1}\left(b_{k}\right)-\alpha^{-1}\left(a_{k}\right)\right\} .
$$

Thus, we obtain

$$
\begin{aligned}
\alpha\left\{\sum_{k=1}^{n} \alpha^{-1}\left(b_{k}\right)-\alpha^{-1}\left(a_{k}\right)\right\} & >\alpha\left(\sum_{k=1}^{n}\left(\alpha^{-1}\left(\mu_{k}\right)-\alpha^{-1}\left(\lambda_{k}\right)-\frac{\alpha^{-1}(\varepsilon)}{2 n}\right)\right) \\
& =\alpha\left\{\sum_{k=1}^{n}\left(\alpha^{-1}\left(\mu_{k}\right)-\alpha^{-1}\left(\lambda_{k}\right)\right)-\frac{\alpha^{-1}(\varepsilon)}{2 n}\right\} \\
& =\sum_{k=1}^{n} \mu_{k}-\lambda_{k}-\frac{\varepsilon}{2}>m_{N} G-\frac{\varepsilon}{2}-\frac{\varepsilon}{2}=m_{N} G-\varepsilon .
\end{aligned}
$$

Theorem 7. Let $F$ be a bounded $\alpha$-closed set. Then, the non-Newtonian measure of $F$ is the greatest lower bound of the measure of all possible $\alpha$-open sets containing $F$.

Proof. Let $\Delta$ be an $\alpha$-open interval containing the set $F$. Then, we have

$$
m_{N} F=m_{N} \Delta \dot{-} m_{N}\left(C_{\Delta}^{F}\right) .
$$

By Theorem 5, we can find an $\alpha$-closed set $\Phi$ such that $\Phi \subset C_{\Delta}^{F}$. By Theorem 6, we have

$$
m_{N} \Phi>m_{N} C_{\Delta}^{F}-\varepsilon
$$

for every $\varepsilon>0$. Let define $G_{0}=C_{\Delta}^{\Phi}$. It is clear that $G_{0}$ is an $\alpha$-open set containing $F$. Also, we have

$$
m_{N} G_{0}=m_{N} C_{\Delta}^{\Phi}=m_{N} \Delta \dot{-} m_{N} \Phi \dot{<} m_{N} \Delta \dot{-} m_{N} C_{\Delta}^{F} \dot{+} \varepsilon
$$


Thus, we get

$$
m_{N} G_{0} \dot{<} m_{N} F \dot{+} \varepsilon
$$

Theorem 8. Let the bounded $\alpha$-closed set $F$ be the union of a finite number of pairwise disjoint $\alpha$-closed sets, i.e. $F=\bigcup_{k=1}^{n} F_{k}$, where $F_{k} \cap F_{l}=\varnothing$ for $k \neq l$. Then

$$
m_{N} F={ }_{N} \sum_{k=1}^{n} m_{N} F_{k}
$$

Proof. Since $F$ is $\alpha$-closed set, we have $\alpha^{-1}(F)=\bigcup_{k=1}^{n} \alpha^{-1}\left(F_{k}\right)$ is closed set. Then, by the properties of Lebesgue measure of bounded closed set in real numbers, we have

$$
m\left(\alpha^{-1}(F)\right)=m\left(\bigcup_{k=1}^{n} \alpha^{-1}\left(F_{k}\right)\right)=\sum_{k=1}^{n} m\left(\alpha^{-1}\left(F_{k}\right)\right)
$$

Thus, we get

$$
m_{N} F=\alpha\left(\sum_{k=1}^{n} m\left(\alpha^{-1}\left(F_{k}\right)\right)\right)=\alpha\left(\sum_{k=1}^{n} \alpha^{-1}\left(\alpha\left(m\left(\alpha^{-1}\left(F_{k}\right)\right)\right)\right)\right)={ }_{N} \sum_{k=1}^{n} m_{N} F_{k}
$$

\section{Competing interests}

The authors declare that they have no competing interests.

\section{Authors' contributions}

All authors have contributed to all parts of the article. All authors read and approved the final manuscript.

\section{References}

[1] M. Grossman, R. Katz, Non-Newtonian calculus, 1st ed., Press, Pigeon, Cove Massachusetts, 1972.

[2] J. Grossman, Meta Calculus, Differantial and integral, 1 st ed., Archimedes foundation, Rockpost Massachusetts, 1981.

[3] A.E. Bashirov, E. Mısırlı Gürpınar, A. Özyapıcı, Multiplicative calculus and its applications, Journal of Mathematical Analysis and Applications, 60, 2725-2737, 2008.

[4] C. Duyar, B. Sağır, O. Oğur, Some basic topolocigal properties on non-Newtonian real line, British Journal of Mathematics and Computer Science 9(4), 300-3007, 2015.

[5] C. Duyar, B. Sağır, Non-Newtonian component of Lebesgue measure in real numbers, Hindawi Journal of Mathematics, Volume 13, Issue 6, 2017.

[6] I. P. Natanson, Theory of function of a real variable, vol. 1, Frederic Ungar Publishing CO., New York, NY, USA, 1964.

[7] Tekin, S. and Başar, F., "Certain sequence spaces over the non-Newtonian complex field", Abstract and Applied Analysis, vol. 2013, pp. 1-11, 2013.

[8] Duyar, C., and Erdogan, M., "On non-Newtonian real number series", IOSR Journal of Mathematics, vol. 12, iss. 6, ver. IV, pp. $34-48,2016$.

[9] Çakmak, A. F. and Başar, F., "Some new results on sequence spaces with respect to non-Newtonian calculus", Journal of Inequalities and Applications, vol. 228, no.1, pp. 1-17, 2012.

[10] Duyar, C., and Erdogan, M., ”Non-Newtonian improper integrals”, Journal of Science and Arts, 18(1), pp. 49-74, 2018. 